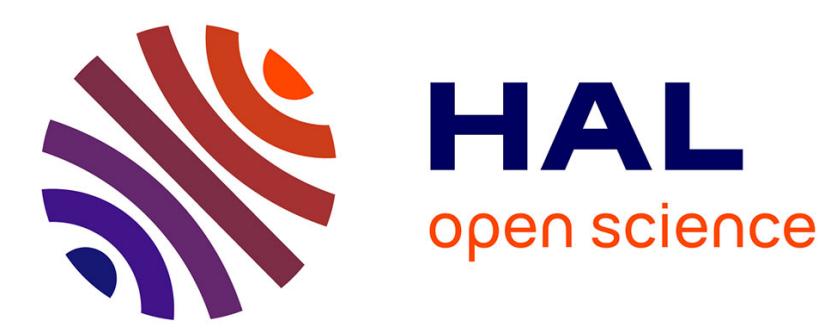

\title{
Approximation of Conditional Preferences Networks "CP-nets" in Possibilistic Logic
}

\author{
Didier Dubois, Souhila Kaci, Henri Prade
}

\section{To cite this version:}

Didier Dubois, Souhila Kaci, Henri Prade. Approximation of Conditional Preferences Networks "CPnets" in Possibilistic Logic. IEEE International Conference on Fuzzy Systems (FUZZ IEEE 2006), Jul 2006, Vancouver, Canada. pp.2337-2342, 10.1109/FUZZY.2006.1682025 . hal-03364220

\section{HAL Id: hal-03364220 \\ https://hal.science/hal-03364220}

Submitted on 5 Oct 2021

HAL is a multi-disciplinary open access archive for the deposit and dissemination of scientific research documents, whether they are published or not. The documents may come from teaching and research institutions in France or abroad, or from public or private research centers.
L'archive ouverte pluridisciplinaire HAL, est destinée au dépôt et à la diffusion de documents scientifiques de niveau recherche, publiés ou non, émanant des établissements d'enseignement et de recherche français ou étrangers, des laboratoires publics ou privés. 


\title{
Approximation of Conditional Preferences Networks "CP-nets" in Possibilistic Logic
}

\author{
Didier Dubois, Souhila Kaci and Henri Prade
}

\begin{abstract}
This paper proposes a first comparative study of the expressive power of two approaches to the representation of preferences: conditional preferences networks (CP-nets) and a logical preference representation framework, namely possibilistic logic. It is shown that possibilistic logic, using a method for handling symbolic priority weights, can always provide complete preorders compatible with the partial $\mathbf{C P}$ net order. Although CP-nets provide an intuitive appealing setting for expressing preferences, possibilistic logic appears to be somewhat more flexible for that purpose.
\end{abstract}

\section{INTRODUCTION}

It is commonly known that preferences are very useful in many real-life problems. Research in AI witnesses a growing importance of the use of preferences if we refer to the different events dedicated to preferences [2], [1], [4], [3].

Preferences between alternatives cannot be generally explicitly expressed because of the large number of possible alternatives or simply because the user is reluctant to express such preferences. Such concerns motivated the development of compact representations of preferences. In fact it is much easier for users to say "I like $p$ more than $q$ " or "if $p$ is true then I prefer $q$ to $\neg q$ ", etc. Compact representations of preferences can be roughly decomposed into three categories: graphical representations (e.g. CP-nets [14], [13]), weighted logical representations (e.g. possibilistic logic [23], penalty logic [19]) and conditional logic of preferences [9], [6], [7], [25], [26], [30].

A compact representation of preferences encodes a partial/total (pre-)order on the set of possible alternatives. Conditional preferences networks (CP-nets for short), which constitute one of the most popular representation formalisms for preference modeling, capture partial orders. $\mathrm{CP}$-nets are directed acyclic graphs based on the expression of conditional ceteris paribus preferences. Possibilistic logic offers another simple representation framework [23] to capture complete preorders encoded by possibility distributions. Preferences are encoded in this framework by means of weighted logical formulas. Lastly, the conditional logic of preferences allows for contextual comparative preferences e.g. if the main dish is fish then I prefer white wine to red wine independently of the other variables [25], [30]. This framework also encodes complete preorders. It has been shown that possibilistic logic can be encoded in the

Didier Dubois: I.R.I.T., 118 route de Narbonne 31062 Toulouse France Souhila Kaci: C.R.I.L., Rue de l'Université SP 1662307 Lens France Henri Prade: I.R.I.T., 118 route de Narbonne 31062 Toulouse France conditional logic of preferences and conversely [12], [21]. Other formalisms stemming from constraint satisfaction networks and answer sets programming have also been adapted to preference modeling [20], [29], [16].

The existence of various formalisms for preference modeling is advantageous in the sense that we have a large choice. However this is also problematic since the more there are formalisms for preference modeling the more we have difficulties to make a choice! Which formalism is the best? On which criteria (complexity, expressiveness) can the formalisms be compared? Is any total/partial (pre)order representable in any corresponding formalism? Are the different formalisms equivalent? Most existing works that addressed some of these questions have mainly focused on complexity issues [18]. However comparing whether the formalisms are equivalent or could be approximated with each other has encountered less interest if we except [20].

The aim of this paper is to go one step further in preference modeling problem by comparing two formalisms, namely CP-nets and possibilistic logic. As previously said possibilistic logic encodes a total preorder while a CP-net encodes a partial order. So these two formalisms cannot be equivalent. The best we can do is to approximate a CP-net in the possibilistic logic setting.

The remainder of this paper is organized as follows. The next section restates the necessary background on CP-nets and possibilistic logic. Section III presents a possibilistic logic approximation of CP-nets. It is established that the approximation is fully compatible with the CP-net in the sense that strict preferences induced by ceteris paribus statements in the CP-net are preserved. In Section IV we show that strict possibilistic preferences turn into strict preferences or incomparabilities in CP-net setting. We show that in the case where priority is given to parent nodes over child nodes, possibilistic strict preferences and CP-net strict preferences coincide. In Section V we consider the extension of CP-nets to TCP-nets and briefly indicate its possibilistic counterpart. We also suggest how the possibilistic framework can be used for handling non-binary preferences referring to many-valued attributes. The concluding remarks outline some directions for future research.

\section{FORMALISMS FOR PREFERENCE MODELING}

Let $V=\left\{X_{1}, \cdots, X_{l}\right\}$ be a set of $l$ variables. Each variable $X_{i}$ takes its values in a domain denoted 
$\operatorname{Dom}\left(X_{i}\right)=\left\{x_{1}^{i}, \cdots, x_{m_{i}}^{i}\right\}$. Let $V^{\prime}$ be a subset of $V$. An assignment of $V^{\prime}$ is the result of assigning a value in $\operatorname{Dom}\left(X_{i}\right)$ to each variable $X_{i}$ in $V^{\prime}$. Asst $\left(V^{\prime}\right)$ is the set of all possible assignments to variables in $V^{\prime}$. In particular $A s s t(V)$, denoted $\Omega$, is the set of all possible assignments of the variables in $V$. Each element in $\Omega$, denoted $\omega$, is called an alternative.

A preorder on $\Omega$ is denoted $\succeq: \omega \succeq \omega^{\prime}$ is interpreted as $\omega$ is at least as preferred as $\omega^{\prime}$. $\omega \succ \omega^{\prime}$ means that $\omega$ is strictly preferred to $\omega^{\prime}$, i.e. $\omega \succeq \omega^{\prime}$ holds but not $\omega^{\prime} \succeq \omega$. $\omega \simeq \omega^{\prime}$ means that $\omega$ and $\omega^{\prime}$ are equally preferred, i.e. both $\omega \succeq \omega^{\prime}$ and $\omega^{\prime} \succeq \omega$ hold. Lastly $\omega \sim \omega^{\prime}$ means that $\omega$ and $\omega^{\prime}$ are incomparable, i.e. neither $\omega \succeq \omega^{\prime}$ nor $\omega^{\prime} \succeq \omega$ hold.

A preorder is complete (or total) if it is connected, i.e. $\forall \omega, \omega^{\prime} \in \Omega$ we have $\omega \succeq \omega^{\prime}$ or $\omega^{\prime} \succeq \omega$.

\section{A. Conditional preference networks}

A conditional preferences network (CP-net for short) [14], [13] is a directed graphical representation of conditional preferences, where nodes represent variables and edges express preference links between them. When there exists a link from $X$ to $Y, X$ is said to be a parent of $Y . P a(X)$ denotes the set of parents of a given node $X$. It determines the user's preferences over possible values of $X$. For the sake of simplicity, we suppose that variables are binary. We also consider acyclic CP-nets in order to ensure the consistency of the CP-net [13].

Preferences are expressed at each node by means of a conditional preference table (CPT for short) such that:

- for root nodes $X_{i}$, the conditional preference table denoted $C P T\left(X_{i}\right)$ provides the preference among $x^{i}$ and its negation $\neg x^{i}$ other things being equal i.e. $\forall y \in$ $\operatorname{Asst}(Y), x^{i} y \succ \neg x^{i} y$ where $Y=V \backslash\left\{X_{i}\right\}$. This is the ceteris paribus assumption.

- For other nodes $X_{j}, C P T\left(X_{j}\right)$ describes the preferences among $x^{j}$ and $\neg x^{j}$ other things being equal given any assignment of $\operatorname{Pa}\left(X_{j}\right)$ i.e. $x^{j} z y \succ \neg x^{j} z y$, $\forall z \in \operatorname{Asst}\left(P a\left(X_{j}\right)\right)$ and $\forall y \in \operatorname{Asst}(Y)$ where $Y=V \backslash\left(\left\{X_{j}\right\} \cup \operatorname{Pa}\left(X_{j}\right)\right)$.

A complete ordering $\succ$, called also preference ranking or preference ordering, satisfies a CP-net $N$ iff it satisfies each conditional preference expressed in $N$. In this case, we say that $\succ$ is consistent with $N$.

Definition 1 (Preference entailment): Let $N$ be a CP-net over a set of variables $V$, and $\omega, \omega^{\prime} \in \Omega$ be any two alternatives. $N$ entails that $\omega$ is strictly preferred to $\omega^{\prime}$, denoted $N \models \omega \succ \omega^{\prime}$ or $\omega \succ_{N} \omega^{\prime}$, iff $\omega \succ \omega^{\prime}$ holds in every preference ordering $\succ$ that satisfies $N$.

Example 1: (borrowed from [13]) Let $N$ be a CP-net presented in Figure 1 modeling preferences on an evening suit. The CP-net is built on three variables $J, P$ and $S$

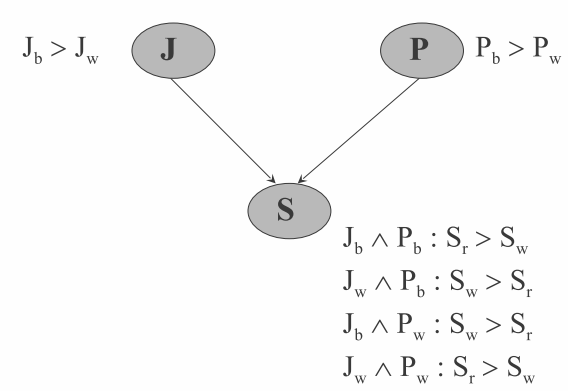

Fig. 1. Evening suit.

representing respectively jacket, pants and shirt. The user has an unconditional preference to black over white for both jacket and pants, while her preference about red or white shirt depends on the combination of jacket and pants's colours: if they have the same colour then she prefers a red shirt otherwise she prefers a white shirt.

An important problem in preference representation is to answer queries about the comparison of alternatives. Let $\omega$ and $\omega^{\prime}$ be two alternatives. Queries consists in asking whether $N \models \omega \succ \omega^{\prime}$ i.e. $\omega$ is preferred to $\omega^{\prime}$, or $N \models \omega^{\prime} \succ \omega$ i.e. $\omega^{\prime}$ is preferred to $\omega$, or $N \forall \omega \succ \omega^{\prime}$ and $N \forall \omega^{\prime} \succ \omega$ i.e. based on knowledge expressed by the CP-net, we are able to prove neither $N \models \omega \succ \omega^{\prime}$ nor $N \models \omega^{\prime} \succ \omega$. Note that CP-nets do not model indifference so two alternatives cannot be equally preferred. We distinguish two types of queries to compare alternatives w.r.t. CP-nets [13]:

- Ordering queries consists in asking whether $N \not \omega^{\prime} \succ$ $\omega$. If this relation holds then this means that there exists a preference ordering $\succ$ consistent with $N$ in which $\omega \succ$ $\omega^{\prime}$ i.e., this relation is consistent with the knowledge expressed by $N$ to prefer $\omega$ over $\omega^{\prime}$. In such a case $\omega$ is said to be consistently orderable over $\omega^{\prime}$ with respect to $N$. Ordering queries check the coherence ordering w.r.t. $N$ and allow thus to produce an ordering consistent with $N$ of any set of alternatives.

- Dominance queries consists in asking whether $N \models \omega \succ \omega^{\prime}$. If this relation holds then $\omega$ is preferred to $\omega^{\prime}$ and we say that $\omega$ dominates $\omega^{\prime}$ w.r.t. $N$.

Example 2: (Cont'd) The preference ordering $J_{b} P_{b} S_{r} \succ$ $J_{b} P_{b} S_{w} \succ J_{w} P_{b} S_{w} \succ J_{b} P_{w} S_{w} \succ J_{w} P_{b} S_{r} \succ J_{b} P_{w} S_{r} \succ$ $J_{w} P_{w} S_{r} \succ J_{b} P_{b} S_{r}$ is consistent with $N$. For example $J_{b} P_{b} S_{w}$ is consistently orderable over $J_{w} P_{b} S_{w}$. Also $J_{w} P_{b} S_{r}$ is consistently orderable over $J_{b} P_{w} S_{r}$. This ensures that we have neither $N \models J_{w} P_{b} S_{w} \succ J_{b} P_{b} S_{w}$ nor $N \models$ $J_{b} P_{w} S_{r} \succ J_{w} P_{b} S_{r}$. However this is not sufficient to say that the reverse holds i.e. $N \models J_{b} P_{b} S_{w} \succ J_{w} P_{b} S_{w}$ and $N \models J_{w} P_{b} S_{r} \succ J_{b} P_{w} S_{r}$. Each preference should hold in all complete orders satisfying $N$. 
There are only four preference orderings consistent with $N$, namely the above one and the followings:

- $J_{b} P_{b} S_{r} \succ J_{b} P_{b} S_{w} \succ J_{b} P_{w} S_{w} \succ J_{w} P_{b} S_{w} \succ$ $J_{w} P_{b} S_{r} \succ J_{b} P_{w} S_{r} \succ J_{w} P_{w} S_{r} \succ J_{w} P_{w} S_{w}$,

- $J_{b} P_{b} S_{r} \succ J_{b} P_{b} S_{w} \succ J_{w} P_{b} S_{w} \succ J_{b} P_{w} S_{w} \succ$ $J_{b} P_{w} S_{r} \succ J_{w} P_{b} S_{r} \succ J_{w} P_{w} S_{r} \succ J_{w} P_{w} S_{w}$,

- $J_{b} P_{b} S_{r} \succ J_{b} P_{b} S_{w} \succ J_{b} P_{w} S_{w} \succ J_{w} P_{b} S_{w} \succ$ $J_{b} P_{w} S_{r} \succ J_{w} P_{b} S_{r} \succ J_{w} P_{w} S_{r} \succ J_{w} P_{w} S_{w}$.

Indeed we have $N \models J_{b} P_{b} S_{w} \succ J_{w} P_{b} S_{w}$ and $N \forall$ $J_{w} P_{b} S_{r} \succ J_{b} P_{w} S_{r}$.

Figure 2 gives the partial order associated with $N$. For the

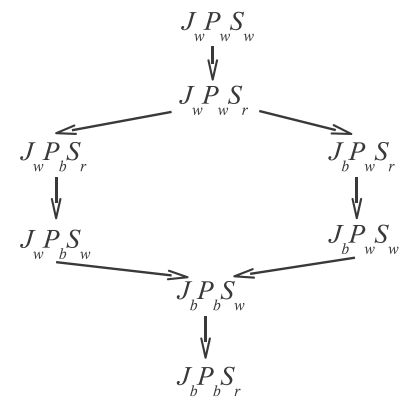

Fig. 2. Partial order induced by $N$.

sake of simplicity we did not represent strict preferences which can be induced by transitivity. An arrow from an alternative $\omega$ to another alternative $\omega^{\prime}$ means that $\omega^{\prime}$ is strictly preferred to $\omega$.

\section{B. Possibilistic logic}

Preferences are encoded in possibilistic logic by means of a set of weighted formulas of the form

$$
\Sigma=\left\{\left(\phi_{i}, \alpha_{i}\right): i=1, \cdots, n\right\},
$$

where $\phi_{i}$ is a propositional formula encoding an agent's goal and $\alpha_{i}$ is a weight representing the priority associated with $\phi_{i}$.

Possibilistic logic [23] is a suitable framework to encode complete preorders. When the "weights" $\alpha_{i}(i=1, \cdots, n)$ belong to a completely ordered scale finite or not, a complete preorder is derived from $\Sigma$ in the following way [23]:

Definition 2: Let $\Sigma$ be a possibilistic base. A complete preorder associated to $\Sigma$, denoted $\succeq_{\Sigma}$, is defined by: $\forall \omega, \omega^{\prime} \in \Omega$,

$$
\omega \succ_{\Sigma} \omega^{\prime} \text { iff } \max \left\{\alpha_{i}: \omega \not \models \phi_{i}\right\}<\max \left\{\alpha_{i}: \omega^{\prime} \not \models \phi_{i}\right\} .
$$

Also $\omega \simeq \omega^{\prime}$ iff $\max \left\{\alpha_{i}: \omega \not \models \phi_{i}\right\}=\max \left\{\alpha_{i}: \omega^{\prime} \not \models \phi_{i}\right\}$.

\section{APPROXIMATION OF CP-NETS IN POSSIBILISTIC LOGIC}

Since CP-nets encode partial orders while possibilistic logic encodes a complete preorder, these two formalisms cannot be equivalent. The best we can do is to approximate $\mathrm{CP}$-nets in possibilistic logic. A faithful approximation of a $\mathrm{CP}-$ net in possibilistic logic consists in preserving all strict preferences induced by the CP-net. This approximation is useful since a weak information about the comparison of two alternatives is sufficient in some applications [13]. Suppose that user's preferences are encoded by means of a CP-net $N$. Let $\omega_{1}$ and $\omega_{2}$ be two alternatives. Then it may be that the worst situation for the user is when $N \models \omega_{2} \succ \omega_{1}$ so she asks whether $N \not \forall \omega_{2} \succ \omega_{1}$. The user will be "relieved" if we ensure that $N \forall \omega_{2} \succ \omega_{1}$ and does not matter whether $N \models \omega_{1} \succ \omega_{2}$ or $\omega_{1}$ and $\omega_{2}$ are incomparable w.r.t $N$. Notice that such a query is simply an ordering query from a CP-net. In this section we show that ordering queries can be encoded in possibilistic logic. Moreover the proposed encoding is a faithful approximation of CP-nets since it recovers all strict preferences induced by ceteris paribus statements in the CP-net. The obtained preorder is indeed a linearization of the partial order induced by the CP-net. The approximation technique follows three steps:

\section{1) Local approximation:}

We associate to each node $X_{i}$ in $N$ a possibilistic base $\Sigma_{X_{i}}$ such that any ceteris paribus statement in $\operatorname{CPT}\left(X_{i}\right)$ is recovered in the preorder associated to $\Sigma_{X_{i}}$. Let $c_{i k}: x \succ x^{\prime}$ be the preferences in $\operatorname{CPT}\left(X_{i}\right)$, where $c_{i k} \in \operatorname{Asst}\left(\operatorname{Pa}\left(X_{i}\right)\right)$. $x$ and $x^{\prime}$ are either equal to $x^{i}$ or $\neg x^{i}$ since we consider binary variables. The following possibilistic base is associated with $X_{i}$ :

$$
\Sigma_{X_{i}}=\left\{\left(\bigvee_{k}\left(c_{i k} \wedge x\right), \alpha_{i}\right)\right\},
$$

where $\alpha_{i}$ is a symbolic weight in the interval $[0,1]$.

The disjunction on $k$ is justified by the fact that each alternative satisfying $c_{i k}$ should satisfy $x$ and not $x^{\prime}$. This guarantees to recover ceteris paribus preferences induced by $C P T\left(X_{i}\right)$.

Example 3: (Cont'd) We have $\Sigma_{J}=\left\{\left(J_{b}, \alpha_{1}\right)\right\}$, $\Sigma_{P}=\left\{\left(P_{b}, \alpha_{1}\right)\right\}^{1}$ and $\Sigma_{S}=\left\{\left(J_{b} P_{b} S_{r} \vee J_{w} P_{b} S_{w} \vee\right.\right.$ $\left.\left.J_{b} P_{w} S_{w} \vee J_{w} P_{w} S_{r}, \beta_{1}\right)\right\}$.

The possibilistic logic base $\Sigma_{X_{i}}$ associated with a node $X_{i}$ recovers all ceteris paribus statements given in $C P T\left(X_{i}\right)$. Formally we write:

Proposition 1: Let $N$ be a CP-net over a set of variables $V=\left\{X_{1}, \cdots, X_{l}\right\}$. Let $\Sigma_{X_{i}}$ be the possibilistic base associated with $C P T\left(X_{i}\right)$ as described above.

\footnotetext{
${ }^{1}$ Since $J$ and $P$ are independent we can give them the same weight $\alpha_{1}$.
} 
Then,

$$
\forall \omega, \omega^{\prime} \in \Omega \text {, if } \omega \succ_{C P T\left(X_{i}\right)} \omega^{\prime} \text { then } \omega \succ_{\Sigma} \omega^{\prime},
$$

where $\succ_{C P T\left(X_{i}\right)}$ denotes the preference given by the node $X_{i}$ in $N$.

Example 4: (Cont'd) Let's consider the base $\Sigma_{J}$. Following Definition 2, we have $\succeq_{\Sigma_{J}}: J_{b} P_{b} S_{r} \simeq$ $J_{b} P_{b} S_{w} \simeq J_{b} P_{w} S_{r} \simeq J_{b} P_{w} S_{w} \succ J_{w} P_{b} S_{r} \simeq$ $J_{w} P_{b} S_{w} \simeq J_{w} P_{w} S_{r} \simeq J_{w} P_{w} S_{w}$. We can check that we recover all ceteris paribus preferences induced by $C P T(J)$.

\section{2) Aggregating parent's bases:}

On the basis of the results of step 1, we use techniques for merging possibilistic knowledge bases [11], [8] to merge the possibilistic bases associated with the parents of each node. All possibilistic bases associated with the parents of a node will be encoded on the same scale $\alpha_{1}>\cdots>\alpha_{n}$.

Definition 3: [11] Let $\Sigma_{1}=\left\{\left(\phi_{i}, \alpha_{i}\right): i=1, \cdots, n\right\}$ and $\Sigma_{2}=\left\{\left(\psi_{j}, \beta_{j}\right): j=1, \cdots, m\right\}$. Let $\oplus$ be a merging operator defined from $[0,1] \times[0,1]$ into $[0,1]$. The result of merging $\Sigma_{1}$ and $\Sigma_{2}$ using $\oplus$ is:

$$
\begin{aligned}
& \Sigma_{\oplus}=\{\left.\left(\phi_{i}, \oplus\left(\alpha_{i}, 0\right)\right)\right\} \cup\left\{\left(\psi_{j}, \oplus\left(0, \beta_{j}\right)\right)\right\} \\
& \cup\left\{\left(\phi_{i} \vee \psi_{j}, \oplus\left(\alpha_{i}, \beta_{j}\right)\right)\right\} .
\end{aligned}
$$

For the purpose of this paper we need a merging operator which satisfies the following properties:

(i) associative,

(ii) 0 is the neutral element,

(iii) 1 is the absorbing element and

(iv) $\oplus(\alpha, \beta)>\max (\alpha, \beta)$ for $(\alpha, \beta) \in] 0,1\left[^{2}\right.$.

(v) $\oplus$ is strictly increasing in both places.

These operators are called strict co-norms [17]. An example of these operators is the probabilistic sum defined by $\oplus(\alpha, \beta)=\alpha+\beta-\alpha \beta$, that we will use in this paper. Indeed $\Sigma_{\oplus}=\Sigma_{1} \cup \Sigma_{2} \cup\left\{\left(\phi_{i} \vee \psi_{j}, \alpha_{i}+\beta_{j}-\alpha_{i} \beta_{j}\right)\right\}$.

A nice property of the strict co-norm operators is that they allow to order the alternatives in the following way. Let $X_{i}$ be a node having $m$ parents. Following this operator, the best alternatives are those which satisfy all $C P T($.$) of P a\left(X_{i}\right)$. Alternatives which are immediately less preferred will be those which satisfy $(m-1) C P T($.$) of P a\left(X_{i}\right)$ and so on. So the least preferred alternatives are those which do not satisfy any $C P T($.$) of P a\left(X_{i}\right)$.

Example 5: (Cont'd) We merge $\Sigma_{J}$ et $\Sigma_{P}$ using the probabilistic sum operator. We get $\Sigma_{J P}=$ $\left.\left\{J_{b} \vee P_{b}, \gamma_{1}\right),\left(J_{b} \wedge P_{b}, \alpha_{1}\right)\right\}$, with $\gamma_{1}>\alpha_{1}$. We have $\succeq_{J P}: J_{b} P_{b} S_{r} \simeq J_{b} P_{b} S_{w} \succ J_{b} P_{w} S_{r} \simeq J_{b} P_{w} S_{w} \simeq$
$J_{w} P_{b} S_{r} \simeq J_{w} P_{b} S_{r} \succ J_{w} P_{w} S_{r} \simeq J_{w} P_{w} S_{w}$.

Indeed we get the expected ordering giving priority to alternatives satisfying more parent nodes, as explained above.

3) Aggregating parent's bases and child's base:

Lastly we combine the possibilistic base associated to each node with the one pertaining to its parents resulting from step 2. We follow the lexicographic principle also called the dictatorship principle. Note that in CP-nets the dictator is the possibilistic base associated to the parent node. This follows from the fact that when two alternatives are incomparable w.r.t. a parent node in CP-nets, preferences associated to a child node may be useful to distinguish between them. In the possibilistic framework this means that when two alternatives are equally preferred w.r.t. the dictator base then they are compared w.r.t. the "non-dictator" base (associated with a child node). The following proposition gives the possibilistic encoding of the lexicographic principle:

Proposition 2: Let $\Sigma_{1}=\left\{\left(\phi_{i}, \alpha_{i}\right): i=1, \cdots, n\right\}$ and $\Sigma_{2}=\left\{\left(\psi_{j}, \beta_{j}\right): j=1, \cdots, m\right\}$ be two possibilistic bases s.t. $\alpha_{1}>\ldots>\alpha_{n}$ and $\beta_{1}>\cdots>\beta_{m}$ (where $\Sigma_{1}$ is the dictator). The result of merging $\Sigma_{1}$ and $\Sigma_{2}$ is:

$$
\begin{aligned}
\Sigma=\{ & \left(\phi_{1} \vee \psi_{1}, \alpha_{11}\right), \cdots,\left(\phi_{1} \vee \psi_{m}, \alpha_{1 m}\right), \\
& \left(\phi_{1}, \alpha_{1}\right), \cdots, \\
& \left(\phi_{n} \vee \psi_{1}, \alpha_{n 1}\right), \cdots,\left(\phi_{n} \vee \psi_{m}, \alpha_{n m}\right), \\
& \left.\left(\phi_{n}, \alpha_{n}\right),\left(\psi_{1}, \beta_{1}\right), \cdots,\left(\psi_{m}, \beta_{m}\right)\right\},
\end{aligned}
$$

where $\alpha_{11}>\cdots>\alpha_{1 m}>\alpha_{1}>\cdots>\alpha_{n 1}>\cdots>$ $\alpha_{n}>\beta_{1}$.

The encoding described in these three steps is a top-down approach namely we start with parent nodes and then propagate the reasoning on child nodes. It is important to note that this encoding holds for every acyclic CP-net.

Proposition 3: Let $N$ be an acyclic CP-net. Let $\Sigma$ be the possibilistic base associated with $N$ and $\succeq_{\Sigma}$ be its associated complete preorder. Then,

$$
\forall \omega, \omega^{\prime} \in \Omega \text {, if } \omega \succ_{N} \omega^{\prime} \text { then } \omega \succ_{\Sigma} \omega^{\prime} .
$$

Example 6: (Cont'd) We merge $\Sigma_{J P}$ and $\Sigma_{S}$ following the lexicographical-based principle, where $\Sigma_{J P}$ is the dictator. We get:

$\Sigma=\left\{\left(J_{b} \vee P_{b} \vee D, \sigma_{1}\right),\left(J_{b} \vee P_{b}, \sigma_{2}\right),\left(\left(J_{b} \wedge P_{b}\right) \vee D, \sigma_{3}\right),\left(J_{b} \wedge\right.\right.$ $\left.\left.P_{b}, \sigma_{4}\right),\left(D, \sigma_{5}\right)\right\}$, with $\sigma_{1}>\sigma_{2}>\sigma_{3}>\sigma_{4}>\sigma_{5}$ and $D=J_{b} P_{b} S_{r} \vee J_{w} P_{b} S_{w} \vee J_{b} P_{w} S_{w} \vee J_{w} P_{w} S_{r}$

The total preorder associated with $\Sigma$ is:

$J_{b} P_{b} S_{r} \succ J_{b} P_{b} S_{w} \succ J_{b} P_{w} S_{w} \simeq J_{w} P_{b} S_{w}$ $\succ J_{w} P_{b} S_{r} \simeq J_{b} P_{w} S_{r} \succ J_{w} P_{w} S_{r} \succ J_{w} P_{w} S_{w}$.

We can check that all strict preferences induced by $N$ are 
preserved. See Figure 2.

Note that the complete preorder obtained in this example recovers all complete orders consistent with $N$ however this is not always the case.

\section{EXTENDING CP-NETS WITH PARENT DOMINANCE PROPERTY}

Let us examine in this section what kind of comparisons of alternatives the possibilistic encoding offers - except the fact already established in the previous section that it preserves all strict preferences induced by the CP-net.

Proposition 4: Let $N$ be an acyclic CP-net and $\Sigma$ be its associated possibilistic logic base. Then,

- $\forall \omega, \omega^{\prime} \in \Omega$, if $\omega \succ_{\Sigma} \omega^{\prime}$ then $\left(\omega \succ_{N} \omega^{\prime}\right.$ or $\left.\omega \sim_{N} \omega^{\prime}\right)$,

- $\forall \omega, \omega^{\prime} \in \Omega$, if $\omega \simeq_{\Sigma} \omega^{\prime}$ then $\omega \sim_{N} \omega^{\prime}$.

It follows from this proposition that the possibilistic encoding does not recover all CP-net incomparabilities. Rather than being a limitation of the possibilistic setting this facts leads us to discuss some of CP-net incomparabilities. In fact following the CP-net of our running example the two alternatives $J_{b} P_{w} S_{r}$ and $J_{w} P_{b} S_{w}$ are incomparable. However $J_{b} P_{w} S_{r}$ falsifies preferences of $P$ and $S$ while $J_{w} P_{b} S_{w}$ falsifies preferences of $J$. The nodes $P$ and $J$ having implicitly the same priority (both are root nodes), this suggests that one may wonder whether $J_{w} P_{b} S_{w}$ should not be given preference over $J_{b} P_{w} S_{r}$. This claim holds also for $J_{w} P_{b} S_{r}$ and $J_{b} P_{w} S_{w}$.

Indeed if we extend traditional CP-net semantics with the requirement that the more an alternative falsifies prioritized nodes, the less preferred it is, then we have the following equivalence between possibilistic logic encoding and such extended CP-nets:

Proposition 5: Let $N$ be an acyclic CP-net where parent nodes dominate child nodes. Let $\Sigma$ be its associated possibilistic logic base. Then, $\forall \omega, \omega^{\prime} \in \Omega$,

- $\omega \succ_{N} \omega^{\prime}$ iff $\omega \succ_{\Sigma} \omega^{\prime}$, and

- $\omega \sim_{N} \omega^{\prime}$ iff $\omega \simeq \omega^{\prime}$.

\section{TCP-NETS AND NON-BINARY PREFERENCES}

TCP-nets [15] have been introduced as an extension of CP-nets for allowing the handling of information about the relative importance of preferences, in order to refine the partial orders induced by the ceteris paribus constraints. For instance if $a \succ \neg a$ and $b \succ \neg b$, the complete order $a b \succ a \neg b \succ \neg a b \succ \neg a \neg b$ can be obtained as soon as priority is given to the first preference, otherwise $a \neg b$ and $\neg a b$ would remain incomparable. TCP-nets allows also for making this type of information context-dependent.

The possibilistic logic approach is based on the idea of expressing relative importance via priority levels. Thus, in possibilistic logic, having both goal $a$ and goal $b$, the first goal being more important writes $\Sigma=\{(a, \alpha),(b, \beta)\}$ when $\alpha$ and $\beta$ are symbolic priority weights such that $\alpha>\beta>0$. This translates in the preorder $a b \succ a \neg b \succ \neg a b \simeq \neg a \neg b$, which may be still refined by adding the further constraint $(a \vee b, \delta)$ with $\delta>\alpha$. If one wants to make the relative importance of $(a, \alpha)$ with respect to $(b, \beta)$ dependent on a context $c$, i.e. having $a \neg b c \succ \neg a b c$ and $\neg a b \neg c \succ a \neg b \neg c$, which expresses that in context $\neg c(b, \beta)$ becomes more important, it amounts to rewriting $\Sigma$ as

$$
\begin{aligned}
& \{(a, \max (\min (\alpha,[c]), \min (\beta,[\neg c]))), \\
& (b, \max (\min (\alpha,[\neg c]), \min (\beta,[c])))\}
\end{aligned}
$$

where $[c]=1$ if $c$ is true and 0 otherwise.

This indicates that the symbolic handling of possibilistic formulas in Section III is also compatible with the introduction of information about the relative importance of preference constraints.

Moreover it is worth pointing out that the possibilistic framework is liable to be extended to the handling of preferences which involve alternatives evaluated by means of non-binary criteria. Indeed, an approach for representing preferences about multifactorial ratings can be proposed in the possibilistic setting. Instead of defining a scale of values and aggregation operations as in classical multiplecriteria approaches, we can express rationality conditions (such that compliance with Pareto ordering) and other generic properties, as well as preferences between specific instances, by means of constraints restricting a complete preordering among tuples of values. The derivation of a single complete preorder can then be based on possibility theory, using the minimal specificity principle, already at work in possibilistic logic, which amounts to preserve indifference between alternatives for which there does not exist a strict preference relation induced by the constraints. This approach looks powerful enough to capture many aggregation modes, even some violating the co-monotonic independence of Choquet integrals [22].

\section{RELATED WORKS AND CONCLUDING REMARKS}

We have shown how CP-net orders can be approximated in the possibilistic logic framework, where preferences are expressed in terms of goals with priority levels. This approximation is equivalent to the one proposed in [20] based on weighted CSP (Constraint Satisfaction Problem). It is also equivalent to the approximation based on answer set optimization [16]. The interest of this result is twofold. First it has a theoretical flavor since it shows that the four settings: possibilistic logic, CP-nets, CSP and answer set optimization are closely related. Second this result shows that preferences can be expressed in different formalisms and thus can benefit from the advantages of each one. Indeed the choice of a particular formalism to use should also be based on other considerations such as complexity, "conciseness", etc [27], [18], [24].

The present work is only a first step towards a thorough comparison of different CPnet-like approaches (including 
developments by [31] or [28]) to the representation of preferences, with the handling in the possibilistic framework.

The investigation of the role of qualitative independence relations [5] in the possibilistic approach should be also further explored.

\section{REFERENCES}

[1] Special issue on Preferences of Computational Intelligence, Computational intelligence, 20(2), 2004.

[2] Preferences in AI and CP: Symbolic Approaches, AAAI02's workshop, 2002.

[3] Advances in Preference Handling, IJCAI05's workshop, 2005.

[4] Preferences: Specification, Inference, Applications, Dagstuhl seminars, 2004.

[5] Benamor and S. Benferhat and D. Dubois and K. Mellouli and H. Prade, A theoretical framework for possibilistic independence in weakly ordered setting, International Journal of Uncertainty Fuzziness and Knowledge-based Systems, 10, 117-155, 2002.

[6] S. Benferhat and D. Dubois and S. Kaci and H. Prade, Bridging logical, comparative and graphical possibilistic representation frameworks, 6th Eur. Conference on Symbolic and Quantitative Approaches to Reasoning and Uncertainty (ECSQARU'01), 422-431, 2001.

[7] S. Benferhat, D. Dubois, S. Kaci and H. Prade, Bipolar Possibilistic Representations, 18th International Conference on Uncertainty in Artifcial Intelligence (UAI'02), 45-52, 2002.

[8] S. Benferhat, D. Dubois, S. Kaci and H. Prade, Possibilistic merging and distance-based fusion of propositional information, 34(1-3), 217252, 2002.

[9] S. Benferhat, D. Dubois and H. Prade, Representing default rules in possibilistic logic, Proceedings of 3rd International Conference of Principles of Knowledge Representation and Reasoning (KR'92), 673684, 1992.

[10] S. Benferhat, D. Dubois and H. Prade, Towards a possibilistic logic handling of preferences, 14(3), 303-317, 2001.

[11] S. Benferhat and D. Dubois and H. Prade and M. Williams, A practical approach to fusing and revising prioritized belief bases, Proceedings of EPIA 99, LNAI $n^{\circ}$ 1695, Springer Verlag, 222-236, 1999.

[12] S. Benferhat and S. Kaci, A possibilistic logic handling of strong preferences, International Fuzzy Systems Association (IFSA'01), 962967,2001

[13] C. Boutilier, R. Brafman, C. Domshlak, H. Hoos and D. Poole, CPnets: A tool for representing and reasoning with conditional ceteris paribus preference statements, Journal of Artificial Intelligence Research, 21, 135-191, 2004.

[14] C. Boutilier, R.I. Brafman, H. Hoos and D. Poole, Reasoning with conditional ceteris paribus preference statements, Proceedings of 15th Conference on Uncertainty in Artificial Intelligence (UAI'99), 71-80, 1999.

[15] R.I. Brafman and C. Domshlak, Introducing Variables Importance Tradeoffs into CP-Nets, Proceedings of 18th Conference on Uncertainty in Artificial Intelligence (UAI'02), 69-76, 2002.

[16] G. Brewka, I. Niemel and M. Truszczynski, Answer Set Optimization, International Joint Conference on Artificial Intelligence (IJCAI'03), 867-872, 2003.

[17] Ep. Klement and R. Mesiar and E. Pap, Triangular norms, Kluwer academic, 2000.

[18] S. Coste-Marquis, J. Lang, P. Liberatore and P. Marquis, Expressive power and succinctness of propositional languages for preference representation, Proceedings of the 9th International Conference on Principles of Knowledge Representation and Reasonning (KR'04), 203212, 2004.

[19] F. Dupin de Saint-Cyr, J. Lang and T. Schiex, Penalty logic and its link with Dempster-Shafer theory, Proceedings of 10th International Conference on Uncertainty in Artificial Intelligence (UAI'94), 204-211, 1994.

[20] C. Domshlak, B. Venable, F. Rossi and T. Walsh, Reasoning about Soft Constraints and Conditional Preferences, International Joint Conference on Artificial Intelligence (IJCAI'03), 215-220, 2003.

[21] D. Dubois, S. Kaci and H. Prade, Ordinal and absolute representations of positive information in possibilistic logic, Proceedings of the International Workshop on Nonmonotonic Reasoning (NMR' 2004), 140-146, 2004.
[22] D. Dubois, S. Kaci and H. Prade, Expressing Preferences From Generic Rules and Examples, Proceedings of 8th European Conference on Symbolic and Quantitative Approaches to Reasoning with Uncertainty (ECSQARU'05), 293-304, 2005.

[23] D. Dubois, J. Lang and H. Prade, Possibilistic logic, In Handbook of Logic in Artificial Intelligence and Logic Programming, D. Gabbay et al., eds, 3, Oxford University Press, 439-513, 1994.

[24] J. Goldsmith, J. Lang, M. Truszczinski and N. Wilson, The computational complexity of dominance and consistency in CP-nets, International Joint Conference on Artificial Intelligence (IJCAI'05), 144-149, 2005.

[25] S. Kaci and L. van der Torre, Algorithms for a Nonmonotonic Logic of Preferences, Eighth European Conference on Symbolic and Quantitative Approaches to Reasoning with Uncertainty (ECSQARU'05), 281-292, 2005.

[26] S. Kaci and L. van der Torre, Non-monotonic Reasoning With Various Kinds of Preferences, IJCAI'05 Multidisciplinary Workshop on Advances in Preference Handling, 2005.

[27] J. Lang, Possibilistic logic: Complexity and Algorithms, In Handbook of Defeasible Reasoning and Uncertainty Management Systems, 5, 179220, 2000.

[28] M. McGeachie and J. Doyle, Efficient Utility Functions for Ceteris Paribus Preferences, 18th National Conference on Artificial Intelligence (AAAI'02), 279-284, 2002.

[29] S. Prestwich, B. Venable, F. Rossi and T. Walsh, Constraint-based Preferential Optimization, National Conference on Artificial Intelligence (AAAI'05), 2005.

[30] L. van der Torre and E. Weydert, Parameters for utilitarian desires in a qualitative decision theory, Applied Intelligence, 14, 285-301, 2001.

[31] N. Wilson, Extending CP-Nets with Stronger Conditional Preference Statements, Proceedings of AAAI 2004, 735-741, 2004. 